\title{
Application Prospect of Cloud Computing in Ubiquity Network
}

\author{
Limin Zhang ${ }^{1, a}$ \\ ${ }^{1}$ Xi'an International University, Xi'an, Shaanxi, 710077
}

\begin{abstract}
Keywords: Cloud Computing; Internet; Application Prospects
\end{abstract}
\begin{abstract}
Ubiquity network refers to the ubiquitous, widespread presence of the Internet. With the development of science and technology, the Internet is gradually improving and improving. For decades, the Internet has basically achieved full coverage in the country. Not only that, it is in all areas of the Internet in the Internet has a very important application status. Cloud computing is one of the world's most advanced science and technology. The emergence of cloud computing accelerates the pace of development is the Internet. So that the Internet is more close to people's lives and become more people benefit the people. This paper mainly introduces the current situation of Ubiquitous network and cloud computing in China, and analyzes the important position and trend of cloud computing in pandemic network development.
\end{abstract}

\section{Introduction}

The current stage of the world is under the influence of the Internet, the formation of a huge global village. From the ancient warfare, to the modern telegraph message, and then today people use a variety of communication software, completely changed people's daily life. Obviously, the pervasive network has become an important part of the grassroots life. Cloud service generation is to meet the people's demand for the network, so that the development of network technology to the foreword of science and technology. Cloud services, virtualization and storage technology to gradually reduce the use of mobile hard disk and $U$ disk, the various network servers have been gradually began to provide cloud services, users will be the data to the cloud more convenient and more secure. Cloud computing makes pan-network services more comprehensive, cloud computing in the ubiquitous network of many applications with epoch-making significance.

\section{The Current Stage of the Domestic Cloud Computing Advanced Science and Technology Application of the Actual Situation}

The increasing popularity of fiber-optic communications and wireless networks, the ever-decreasing price of information storage, and the development of Internet-related software to drive the overall development of cloud computing. The current world IT industry in cloud computing advanced science and technology, including cloud storage, virtualization, large data management, network platform management and other aspects of technology. In the cloud service, not the network provider to provide services, the standard is also different. In the current phase of the pan-network, network providers focus on IaaS and PaaS. IaaS provides the tools needed to calculate, store, network, and build applications as a service to users, enabling users to access IT infrastructure on demand, with core content for virtualization, and virtualization of servers, storage, and networks; PaaS Distributed software development, testing and deployment environment as a service, through the Internet to provide users [1]. Cloud computing enriches the content of the pervasive network, adding many new features to the Ubiquitous network, and making outstanding contributions to the construction of network security. Cloud storage has changed the habits of people in the past to store information, so that information storage toward the non-media aspects of the transition and development.

\section{The Characteristics of the Universal Network}

Ubiquitous networks are widely used in various fields. The current stage of pan-network 
coverage is very extensive. Not only in people's daily life has an important application in all countries in the world also plays a key role. It Includes environmental protection projects and public infrastructure construction and so on. In this field is not professional communications, telecommunications and other business areas, still need to promote these areas of the Internet continue to improve and develop. Any development of today's society has been inseparable from the Internet to promote the role. The Internet has brought people to an informationized world. At this stage, all areas want to get better development, without deepening the development of the Internet within the industry. It can be seen in the wide application of the network, the impact of large.

The existence of network heterogeneous characteristics. The heterogeneity of the Internet is mainly manifested in the following aspects. First, from the tip network, the access network to the core network may use different communication protocols, communication links, such as IP network and non-IP sensor networks, wireless networks to wired networks [2]. Second, there are a variety of domestic mobile communication networks and wireless facilities. Such as 2G, 3G, 4G network, wireless WiFi, Bluetooth and so on. Third, there are various types of wired access links, such as xDSL, xPON, Ethernet, Cable and private communications systems [3]. Fourth, there are a large number of private networks in all areas, and the business is not transparent. Fifth, the difference between addressing and routing, authentication mode, QoS capability, service configuration capability and service system interface between different networks [4]. These are all heterogeneous features of the network.

Ubiquitous network has a large number of service objects. Here the service object is not only refers to the ordinary sense of the people, but also includes the existence of the objective world of material. This increases the number of users in the network in a straight line. And, all kinds of service objects are carrying huge information resources, which led to information in the collection, transmission, storage, processing, when the ubiquitous network to bring a certain impact. Cloud computing in the ubiquitous network of applications just makes up for the social needs of the pan-network caused by the impact of the lack of. So that the pan in the network under normal operation to serve more objects, to provide more quality services. Over time and picnics in the network has more and more clients.

\section{Cloud Computing Contains the Main Technology}

Virtualization Technology. One of the core technologies of cloud computing is virtualization technology. Virtualization technology enables key technologies that fully integrate and efficiently use a variety of computing and storage resources [5]. Virtualization science and technology support the normal operation of cloud computing. Through the virtualization of advanced technology, cloud computing can not have any relationship between the application of the deployment of each environment and physical platform through the virtual platform for management, expansion, migration, backup, all operations are completed through the virtualization level [6]. Virtualization of advanced technology just in line with the development of the characteristics and needs of the network, can promote the development of pandemic forward innovation.

The Data Storage Technology. Cloud services have unique storage technology. Can help users to a large number of information resources to the Internet opened up by the virtual space. It cloud storage to achieve the information resources without media storage. So that people can be anytime, anywhere through the Internet to retrieve the existence of cloud information resources. So that people access to storage resources more convenient and easy way. Domestic and foreign network server vendors are developing their own cloud storage services and the approximate type of basic, the user can register their own account on each server and password management of their own storage of information resources. This ensures that information is stored in a relatively secure environment. The development of cloud storage technology has led to the development of various fields. Such as aerospace industry, news industry, power industry and so on.

The Technology that Manages Large Amounts of Data. Computer and network development of advanced science and technology to a certain level, leading to the current Internet there have been a lot of information on the data. Cloud computing can be achieved through the effective 
management of large amounts of data. In the cloud computing management system, the usual data size must reach the TB level or higher level of the pet. It achieves rapid and effective management of massive data. Such as Google's Web page query, to quickly get the information users want the information resources to achieve effective and manage the management.

Management of Network Platform Technology. Cloud computing has a large amount of data resources, a number of network-related servers there are many, it has a different structure, and were distributed in different geographical location of the relevant data center. How to manage the tens of thousands of applications running at high speed in cloud services, ensuring that the entire network system can provide 24 hours of service around the day is a huge challenge for the relevant departments at this stage. Cloud computing through its management platform for intelligent management, is the server together to cooperate, timely detection of failure, repair system to protect the user's needs for the network to get the most satisfaction.

\section{The Network of Cloud Computing Advanced Science and Technology Application Prospects}

Continue to expand the network cloud service system open efforts. At this stage of the network server operators to the majority of grassroots people to a certain extent, open the cloud services. Most operators have a comprehensive and detailed description of the resources in their own managed areas, creating a well-organized network cloud environment that makes the sharing of data resources more affordable. Users can also according to their own different actual situation, and operators to communicate, pay for operators to provide more cloud services. This kind of open internet cloud service satisfies the demand for data resources in the background of the current data and information.

To create a relatively stable and secure network environment. Cloud computing continues to create a more secure Internet operating environment for pan-network systems. Cloud computing to build a server between the cloud and the cloud between the connection channel and you can achieve for the pan-network in the parallel management. For people collect information, it storage information, processing information and other projects to provide a relatively safe Internet environment. Cloud services can effectively avoid the network data resources leaked or lost the phenomenon. Information stored in the Internet to build the virtual storage space, the use of cloud computing advanced technology for scientific data and platform management, for the pan-network to create a relatively secure Internet environment.

With automatically selected operation function. Automatic selection is characterized by the user can through their own actual needs, in the cloud automatically select their own needs data resources. This can effectively improve the efficiency of users and cloud data resources on the use of efficiency. In the ubiquitous network in the continuous application of cloud computing advanced science and technology, you can gradually reduce the automatic selection of data resources and enhance the accuracy of automatic access to cloud content.

Enriched the content of the pan-network structure. The widespread use of cloud computing in pervasive cyberspace enriches the content of the pervasive network. The cloud computing is for the ubiquitous network to bring cloud data storage, data management, network platform management and other new content. So that the scope of the universal network services in more and more widely, with more and more full functionality. Whether it is cloud storage technology or data and platform for the relevant management, are cloud computing in the ubiquitous network of important applications. With the cloud computing continue to move forward in depth, the structure of the content in the network will be more rich and comprehensive.

\section{Conclusion}

The innovation and development of cloud computing directly affect the speed of the development of the pervasive network. The emergence of the Internet has important historical value and significance, want to make the pan-network has been in a leading position in science and technology, we must always ensure that the technical aspects of continuous innovation. Cloud computing in the 
Internet in the important application is to achieve a pan-network innovation and development. So that the ubiquitous network has a comprehensive open cloud service system, creating a more secure network environment, so that the Internet reached a higher level of intelligent level. Cloud computing enriches the content of the ubiquitous network structure, expanding the Internet has the function, so that the application of the Internet in various fields have been more far-reaching impact, to promote the balanced development of various fields.

\section{Acknowledgements}

A project supported by scientific research fund of Shaanxi provincial education department in 2016 (No. 16JK2176);

\section{References}

[1] Shi Jinliang. Analysis of cloud computing technology in the application of radio and television networks [J]. China Media Technology, 2012, 06: 122-123.

[2] Liu Dake, Li Li. Application of cloud computing technology in early childhood education [J]. Henan Science and Technology, 2012, 21: 64-65.

[3] Zhang Jisheng. Cloud computing technology in the power system application [J]. Modern building electrical, 2011, 04: 8-11.

[4] Zhao Guangcai, Zhang Xueping. Cloud computing technology analysis and its prospects [J]. Electronic Design Engineering, 2011, 22: 4-7 +10.

[5] Han Xinzhou. Network security technology and prospect analysis based on cloud computing [J]. Journal of Yantai Vocational College, 2016, 02: 90-92.

[6] Zhou Wen, Li Feng. Cloud computing technology and mobile cloud foreground [J]. Information Systems Engineering, 2013, 09: 25-28 